Bulletin of the Section of Logic

Volume 51/2 (2022), pp. 197-205

https://doi.org/10.18778/0138-0680.2022.01

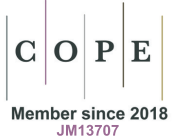

Lew Gordeev

E. Hermann Haeusler

\title{
PROOF COMPRESSION AND NP VERSUS PSPACE II: ADDENDUM
}

\begin{abstract}
In our previous work we proved the conjecture NP $=$ PSPACE by advanced proof theoretic methods that combined Hudelmaier's cut-free sequent calculus for minimal logic (HSC) with the horizontal compressing in the corresponding minimal Prawitz-style natural deduction (ND). In this Addendum we show how to prove a weaker result $\mathbf{N P}=\mathbf{c o N P}$ without referring to HSC. The underlying idea (due to the second author) is to omit full minimal logic and compress only "naive" normal tree-like ND refutations of the existence of Hamiltonian cycles in given non-Hamiltonian graphs, since the Hamiltonian graph problem in NPcomplete. Thus, loosely speaking, the proof of $\mathbf{N P}=\mathbf{c o N P}$ can be obtained by HSC-elimination from our proof of NP = PSPACE.
\end{abstract}

Keywords: Graph theory, natural deduction, computational complexity.

\section{Introduction}

Recall that in $[2,3]$ we proved that intuitionistically valid purely implicational formulas $\rho$ have dag-like ND proofs $\partial$ whose weights (= the total numbers of symbols) are polynomial in the weights $|\rho|$ of $\rho$. $\partial$ were defined by a suitable two-fold horizontal compression of the appropriate tree-like ND $\partial_{1}$ obtained by standard conversion of basic tree-like HSC proofs $\pi$ existing by the validity of $\rho$. We observed that the height and the total weight of distinct formulas occurring in $\left(\pi\right.$, and hence also) $\partial_{1}$ are both

Presented by: Peter Schroeder-Heister

Received: December 1, 2020

Published online: January 07, 2022

(C) Copyright by Author(s), Łódź 2022

(C) Copyright for this edition by Uniwersytet Łódzki, Łódź 2022 
polynomial in $|\rho|$. From this we inferred that the compressed dag-like ND proofs $\partial$ are weight-polynomial in $|\rho|$. Moreover, it is readily seen that the latter conclusion holds true for any tree-like ND $\partial^{\prime}$ with the polynomial upper bounds on the height and total weight of distinct formulas used. We just arrived at the following Theorem 1.3 , where $\mathrm{NM}_{\rightarrow}$ is standard purely implicational ND for minimal logic (see also Appendix and [3] for more details).

Definition 1.1. Tree-like $\mathrm{NM}_{\rightarrow}$-deduction $\partial$ with the root-formula (= conclusion) $\rho$ is called polynomial if its weight (= total number of symbols) is polynomial in the weight of conclusion, $|\rho| . \partial$ is called quasi-polynomial if the height of $\partial$ plus total weight of distinct formulas occurring in $\partial$ is polynomial in $|\rho|$.

Definition 1.2. A given (tree- or dag-like) $\mathrm{NM}_{\rightarrow}$-deduction is called a proof of its root-formula $\rho$ iff every maximal thread connecting $\rho$ with a leaf $\alpha$ is closed, i.e. it contains a "discharging" $(\rightarrow I)$ with conclusion $\alpha \rightarrow \beta$, for some $\beta$.

THEOREM 1.3. In $\mathrm{NM}_{\rightarrow}$, any quasi-polynomial tree-like proof of $\rho$ is compressible into a polynomial dag-like proof of $\rho$.

Now let $P$ be a NP-complete problem and suppose that $\rho$ is valid iff $P$ has no positive solution. From the existence of a tree-like ND proof $\partial^{\prime}$ as above we'll infer the existence of a polynomial dag-like ND proof $\partial$ of $\rho$, which will eventually imply $\mathbf{N P}=\mathbf{c o N P}$. In particular, let $P$ be the Hamiltonian Graph Problem. For any graph $G$ consider purely implicational formula $\rho$ expressing in standard way that $G$ has no Hamiltonian cycles. Suppose that the canonical proof search in $\mathrm{NM}_{\rightarrow}$ yields a normal tree-like proof $\partial^{\prime}$ of $\rho$ whose height is polynomial in $|G|$ (and hence in $|\rho|$ ), provided that $G$ is non-Hamiltonian. Since normal ND proofs satisfy the subformula property, such $\partial^{\prime}$ will obey the requested polynomial upper bounds in question, and hence the weight of its horizontal dag-like compression $\partial$ will be polynomially bounded, as desired. That is, we argue as follows.

Lemma 1.4. Let $P$ be the Hamiltonian graph problem and suppose that purely implicational formula $\rho$ express in standard way that a given graph $G$ has no Hamiltonian cycles. There exists a quasi-polynomial normal treelike proof of $\rho$ in $\mathrm{NM}_{\rightarrow}$ whose height is polynomial in $|G|$ (and hence $|\rho|$ ), provided that $G$ is non-Hamiltonian. 
Recall that polynomial ND proofs (whether tree- or dag-like) have timepolynomial certificates ([3]: Appendix), while the non-hamiltoniancy of simple and directed graphs is coNP-complete. Hence Theorem 1.3 yields

Corollary 1.5. $\mathbf{N P}=\mathbf{c o N P}$ holds true.

This argument does not refer to sequent calculus. Summing up, in order to complete our HSC-free proof of $\mathbf{N P}=\mathbf{c o N P}$ it will suffice to prove Lemma 1.4. This will be elaborated in the rest of the paper.

\section{Hamiltonian problem}

Consider a simple ${ }^{1}$ directed graph $G=\left\langle V_{G}, E_{G}\right\rangle, \operatorname{card}\left(V_{G}\right)=n$. A Hamiltonian path (or cycle) in $G$ is a sequence of nodes $\mathcal{X}=v_{1} v_{2} \ldots v_{n}$, such that, the mapping $i \mapsto v_{i}$ is a bijection of $[n]=\{1, \cdots, n\}$ onto $V_{G}$ and for every $0<i<n$ there exists an edge $\left(v_{i}, v_{i+1}\right) \in E_{G}$. The (decision) problem whether or not there is a Hamiltonian path in $G$ is known to be NP-complete (cf. e.g. [1]). If the answer is YES then $G$ is called Hamiltonian. In order to verify that a given sequence of nodes $\mathcal{X}$, as above, is a Hamiltonian path it will suffice to confirm that:

1. There are no repeated nodes in $\mathcal{X}$,

2. No element $v \in V_{G}$ is missing in $\mathcal{X}$,

3. For each pair $\left\langle v_{i}, v_{j}\right\rangle$ in $\mathcal{X}$ there is an edge $\left(v_{i}, v_{j}\right) \in E_{G}$.

It is readily seen that the conjunction of $1,2,3$ is verifiable by a deterministic TM in $n$-polynomial time. Consider a natural formalization of these conditions (cf. e.g. [1]) in propositional logic with one constant $\perp$ (falsum) and three connectives $\wedge, \vee, \rightarrow($ as usual $\neg F:=F \rightarrow \perp)$.

Definition 2.1. For any $G=\left\langle V_{G}, E_{G}\right\rangle, \operatorname{card}\left(V_{G}\right)=n>0$, as above, consider propositional variables $X_{i, v}, i \in[n], v \in V_{G}$. Informally, $X_{i, v}$ should express that vertex $v$ is visited in the step $i$ in a path on $G$. Define propositional formulas $A-E$ as follows and let $\alpha_{G}:=A \wedge B \wedge C \wedge D \wedge E$.

${ }^{1} \mathrm{~A}$ simple graph has no multiple edges. For every pair of nodes $\left(v_{1}, v_{2}\right)$ in the graph there is at most one edge from $v_{1}$ to $v_{2}$. 
1. $A=\bigwedge_{v \in V}\left(X_{1, v} \vee \ldots \vee X_{n, v}\right)$ (: every vertex is visited in $\left.X\right)$.

2. $B=\bigwedge_{v \in V} \bigwedge_{i \neq j}\left(X_{i, v} \rightarrow\left(X_{j, v} \rightarrow \perp\right)\right)$ (: there are no repetitions in $X$ ).

3. $C=\bigwedge_{i \in[n]} \bigvee_{v \in V} X_{i, v}$ (: at each step at least one vertex is visited).

4. $D=\bigwedge_{v \neq w} \bigwedge_{i \in[n]}\left(X_{i, v} \rightarrow\left(X_{i, w} \rightarrow \perp\right)\right)$ (: at each step at most one vertex is visited).

5. $E=\bigwedge_{(v, w) \notin E} \bigwedge_{i \in[n-1]}\left(X_{i, v} \rightarrow\left(X_{i+1, w} \rightarrow \perp\right)\right)$ (: if there is no edge from $v$ to $w$ then $w$ can't be visited immediately after $v)$.

Thus $G$ is Hamiltonian iff $\alpha_{G}$ is satisfiable. Denote by $S A T_{C l a}$ the set of satisfiable formulas in classical propositional logic and by $T A U T_{\text {Int }}$ the set of tautologies in intuitionistic propositional logic. Then the following conditions hold: (1) $G$ is non-Hamiltonian iff $\alpha_{G} \notin S A T_{C l a},(2) G$ is nonHamiltonian iff $\neg \alpha_{G} \in T A U T_{C l a}$, (3) $G$ is non-Hamiltonian iff $\neg \alpha_{G} \in$ $T A U T_{\text {Int }}$. Glyvenko's theorem yields the equivalence between (2) and (3). Hence $G$ is non-Hamiltonian iff there is an intuitionistic proof of $\neg \alpha_{G}$. Such proof is called a certificate for the non-hamiltoniancy of G. [7] (also [4]) presented a translation from formulas in full propositional intuitionistic language into the purely implicational fragment of minimal logic whose formulas are built up from $\rightarrow$ and propositional variables. This translation employs new propositional variables $q_{\gamma}$ for logical constants and complex propositional formulas $\gamma$ (in particular, every $\alpha \vee \beta$ and $\alpha \wedge \beta$ should be replaced by $q_{\alpha \vee \beta}$ and $q_{\alpha \wedge \beta}$, respectively) while adding implicational axioms stating that $q_{\gamma}$ is equivalent to $\gamma$. For any propositional formula $\gamma$, let $\gamma^{\star}$ denote its translation into purely implicational minimal logic in question. Note that size $\left(\gamma^{\star}\right) \leq(\operatorname{size}(\gamma))^{3}$. Now let $\gamma:=\neg \alpha_{G}$. So $\gamma \in T A U T_{\text {Int }}$ iff $\gamma^{\star}$ is provable in the minimal logic. Moreover, it follows from [7], [4] that for any normal intuitionistic ND proof $\partial$ of $\gamma$ there is a normal proof $\partial_{\rightarrow}$ of $\gamma^{\star}$ in the corresponding ND system for minimal logic, $\mathrm{NM}_{\rightarrow}$, such that height $\left(\partial_{\rightarrow}\right)=\mathcal{O}($ height $(\partial))$. Thus in order to prove Lemma 1.4 it will suffice to establish

Claim 2.2. $G$ is non-Hamiltonian iff there exists a normal intuitionistic tree-like ND proof of $\alpha_{G} \rightarrow \perp$, i.e. $\neg \alpha_{G}$, whose height is polynomial in $n$. 


\subsection{Proof of Claim 2.2}

The sufficiency easily follows from the soundness of ND. Consider the necessity. In the sequel we suppose that a non-Hamiltonian graph $G$ is fixed and $\alpha_{G}=A \wedge B \wedge C \wedge D \wedge E$ (cf. Definition 2.1). Let $p$ : $\{1, \ldots, n\} \mapsto V_{G}$ be any sequence of nodes from $V_{G}$ of the length $n$ and let $\mathcal{X}_{p}:=\left\{X_{1, p[1]}, \cdots, X_{n, p[n]}\right\}$ be corresponding set of propositional variables. $\mathcal{X}_{p}$ and $p$ represent a path in $G$ that starts by visiting vertex $p[1]$, encoded by $X_{1, p[1]}$, followed by $p[2]$, encoded by $X_{2, p[2]}$, etc., up to $p[n]$ encoded by $X_{n, p[n]}$. Since $G$ is non-Hamiltonian, $\mathcal{X}_{p}$ is inconsistent with $\alpha_{G}$.

Lemma 2.3. For any $p$ and $\mathcal{X}_{p}$ as above there is a normal intuitionistic tree-like $N D \Pi_{p}$ with conclusion $\perp$, assumptions from $\mathcal{X}_{p} \cup\left\{\alpha_{G}\right\}$ and height $\left(\Pi_{p}\right)=\mathcal{O}\left(n^{2}\right)$ :

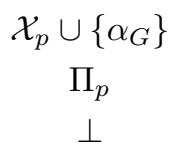

Proof: $\Pi_{p}$ is defined as follows. Since $G$ is non-Hamiltonian, we observe that at least one of the conditions 1, 3 to be a Hamiltonian path (see above in $\S 2$ ) fails for $\mathcal{X}_{p}$. Hence at least one of the following is the case.

There are repeated nodes. There are $1 \leq i<j \leq n$, such that $p[i]=p[j]=$ $v \in V_{G}$; let $i<j$ be the least such pair. Consider a deduction $\Gamma_{p}$ :

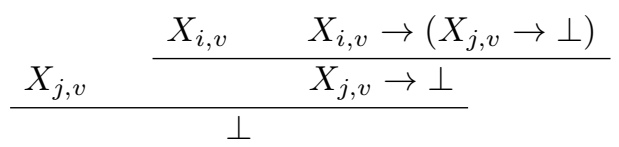

of $\perp$ from $X_{i, v}, X_{j, v}$ and $X_{i, v} \rightarrow\left(X_{j, v} \rightarrow \perp\right)$. Since $\left\{X_{i, v}, X_{j, v}\right\} \subset$ $\mathcal{X}_{p}$, the assumption $X_{i, v} \rightarrow\left(X_{j, v} \rightarrow \perp\right)$ is a component of the conjunction $B$ from $\alpha_{G}$. So let $\Delta_{p}$ be a chain of $\wedge$-elimination rules deducing $X_{i, v} \rightarrow\left(X_{j, v} \rightarrow \perp\right)$ from $\alpha_{G}$. Now let $\Pi_{p}$ be the corresponding 
concatenation $\Delta_{p} \circ \Gamma_{p}$ deducing $\perp$ from $\left\{X_{i, v}, X_{j, v}, \alpha_{G}\right\} \subset \mathcal{X}_{p} \cup\left\{\alpha_{G}\right\}$. Clearly $\Pi_{p}$ is normal and height $\left(\Pi_{p}\right)=\mathcal{O}\left(n^{2}\right)$.

There is a missing edge. There is $1 \leq i<n$, such that $p[i]=v \in V_{G}$, $p[i+1]=w \in V_{G}$ and $(v, w) \notin E_{G}$; Let $i$ be the least such number. Consider a deduction $\Gamma_{p}$ :

$$
\begin{gathered}
X_{i+1, w} \quad \frac{X_{i, v} \quad X_{i, v} \rightarrow\left(X_{i+1, w} \rightarrow \perp\right)}{X_{i+1, w} \rightarrow \perp} \\
\perp
\end{gathered}
$$

of $\perp$ from $X_{i, v}, X_{i+1, w}$ and $X_{i, v} \rightarrow\left(X_{i+1, w} \rightarrow \perp\right)$. Since $\left\{X_{i, v}, X_{i+1, w}\right\}$ $\subset \mathcal{X}_{p}$ and $(v, w) \notin E_{G}$, the assumption $X_{i, v} \rightarrow\left(X_{i+1, w} \rightarrow \perp\right)$ is a component of the conjunction $E$ from $\alpha_{G}$. So let $\Delta_{p}$ be a chain of $\wedge$-elimination rules deducing $X_{i, v} \rightarrow\left(X_{i+1, w} \rightarrow \perp\right)$ from $\alpha_{G}$. Now let $\Pi_{p}$ be the corresponding concatenation $\Delta_{p} \circ \Gamma_{p}$ deducing $\perp$ from $\left\{X_{i, v}, X_{i+1, w}, \alpha_{G}\right\} \subset \mathcal{X}_{p} \cup\left\{\alpha_{G}\right\}$. Clearly $\Pi_{p}$ is normal and height $\left(\Pi_{p}\right)=\mathcal{O}\left(n^{2}\right)$.

In the sequel for the sake of brevity we let $V_{G}=\{1, \cdots, n\}$. Now consider the deductions $\Pi_{p}^{i}, 1 \leq i \leq n$, in the extended ND that includes standard $n$-ary $\vee$-elimination rules. $\Pi_{p}^{i}$ are defined by recursion on $i$ using (in the initial case $i=1$ ) the $\Pi_{p(1 / k)}$ from the last lemma, where sequences $p(-j):\{1, \ldots, n\} \mapsto V_{G} \cup\{0\}$ and $p(j / k):\{1, \ldots, n\} \mapsto V_{G}$ are defined by

$$
p(-j)[k]:=\left\{\begin{array}{ll}
p[k], & \text { if } \\
0, & \text { else }
\end{array} \quad k=j\right.
$$

and

$$
p(j / k):= \begin{cases}p[k], & \text { if } \quad k=j, \\ p[j], & \text { else. }\end{cases}
$$

So let

$$
\begin{aligned}
& \Pi_{p}^{1}= \\
& \mathcal{X}_{p(-1)} \cup\left\{\alpha_{G}\right\},\left[X_{1, v_{1}}\right] \quad \mathcal{X}_{p(-1)} \cup\left\{\alpha_{G}\right\},\left[X_{n, v_{n}}\right] \\
& \Pi_{p(1 / 1)} \quad \Pi_{p(1 / n)} \\
& \begin{array}{llll}
X_{1, v_{1}} \vee \cdots \vee X_{1, v_{n}} & \perp & \cdots & \perp
\end{array},
\end{aligned}
$$




$$
\begin{aligned}
& \Pi_{p}^{j+1}:= \\
& \mathcal{X}_{p(-(j+1))} \cup\left\{\alpha_{G}\right\},\left[X_{j+1, v_{1}}\right] \quad \mathcal{X}_{p(-(j+1))} \cup\left\{\alpha_{G}\right\},\left[X_{j+1, v_{n}}\right] \\
& \Pi_{p((j+1) / 1)}^{j} \quad \Pi_{p((j+1) / n)}^{i} \\
& \begin{array}{lllll}
X_{j+1, v_{1}} \vee \cdots \vee X_{j+1, v_{n}} & \perp & & \cdots & \perp \\
\hline & \perp &
\end{array} .
\end{aligned}
$$

Thus for $i=n$ we obtain.

$$
\begin{aligned}
& \Pi_{p}^{n}= \\
& \mathcal{X}_{p(-(n-1))} \cup\left\{\alpha_{G}\right\},\left[X_{n, v_{1}}\right] \quad \mathcal{X}_{p(-(n-1))} \cup\left\{\alpha_{G}\right\},\left[X_{n, v_{n}}\right] \\
& \Pi_{p(n / 1)}^{n-1} \quad \Pi_{p(n / n)}^{n-1} \\
& \begin{array}{lllll}
X_{n, v_{1}} \vee \cdots \vee X_{n, v_{n}} & \perp & & \cdots & \perp
\end{array} .
\end{aligned}
$$

Lemma 2.4. For any $p:\{1, \ldots, n\} \mapsto V_{G}, \Pi_{p}^{n}$ is a normal intuitionistic tree-like deduction with conclusion $\perp$ and (the only) open assumption $\alpha_{G}$ in the extended $N D$ in question Moreover, height $\left(\Pi_{p}^{n}\right)=\mathcal{O}\left(n^{2}\right)$.

Proof: This easily follows from Lemma 2.4 by induction on $n$.

Now let $\Pi:=\Pi_{I d}^{n}$ where $I d:\{1, \ldots, n\} \mapsto V_{G}$ is the identity $\operatorname{Id}[i]:=i$. Denote by $\widehat{\Pi}$ the canonical tree-like embedding of $\Pi$ into basic intuitionistic ND with plain (binary) $\vee$-eliminations that is obtained by successive unfolding of the $n$-ary $\vee$-elimination rules with premises $X_{j, v_{1}} \vee \cdots \vee X_{j, v_{n}}$ involved. Note that height $(\widehat{\Pi})=\mathcal{O}\left(n^{3}\right)$. Moreover let $\partial$ denote $\widehat{\Pi}$ followed by the introduction of $\alpha_{G} \rightarrow \perp$ :

$$
\begin{aligned}
& {\left[\alpha_{G}\right] } \\
& \widehat{\Pi} \\
& \alpha_{G} \quad \perp \\
& \hline \alpha_{G} \rightarrow \perp
\end{aligned}
$$

Corollary 2.5. $\partial$ is a normal intuitionistic tree-like ND proof of $\alpha_{G} \rightarrow \perp$ whose height is polynomial in $n$, as required. 


\section{Appendix: More on Theorem 1.3}

Theorem 1.3 (cf. Introduction). In standard ND for purely implicational minimal logic, $\mathrm{NM}_{\rightarrow}$, any quasi-polynomial tree-like proof $\partial$ of $\rho$ is compressible into a polynomial dag-like proof $\partial^{*}$ of $\rho$.

Proof SketCH ${ }^{2}$ : The mapping $\partial \hookrightarrow \partial^{*}$ is obtained by a two-folded horizontal compression $\partial \hookrightarrow \partial^{b} \hookrightarrow \partial^{*}$, where $\partial^{b}$ is a polynomial dag-like deduction in $\mathrm{NM}_{\rightarrow}^{b}$ that extends $\mathrm{NM}_{\rightarrow}$ by a new separation rule $(S)$

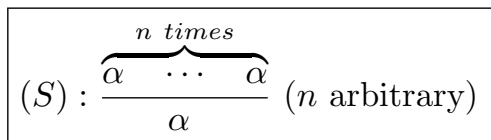

whose identical premises are understood disjunctively: "if at least one premise is proved then so is the conclusion" (in contrast to ordinary inferences: "if all premises are proved then so are the conclusions"). The notion of provability in $\mathrm{NM}_{\rightarrow}^{b}$ is modified accordingly such that proofs are locally correct deductions assigned with appropriate sets of threads that are closed and satisfy special conditions of local coherency. Now $\partial^{b}$ arises from $\partial$ by ascending (starting from the root) merging of different occurrences of identical formulas occurring on the same level, followed by inserting instances of $(S)$ instead of resulting multipremise inferences. Corresponding locally coherent threads in $\partial^{b}$ are inherited by the underlying (closed) threads in $\partial$ (in contrast to ordinary local correctness, the local coherency is not verifiable in polynomial time, as the total number of threads in question might be exponential in $|\rho|$ ). A desired "cleansed" $\mathrm{NM}_{\rightarrow}$-subdeduction $\partial^{*} \subset \partial^{b}$ arises by collapsing $(S)$ to plain repetitions

$$
(R): \frac{\alpha}{\alpha}
$$

with respect to the appropriately chosen premises of $(S)$. The choice is made non-deterministically using the set of locally coherent threads in $\partial^{b}$.

\footnotetext{
${ }^{2}$ See [3] for more details.
} 


\section{References}

[1] S. Arora, B. Barak, Computational Complexity: A Modern Approach, Cambridge University Press (2009).

[2] L. Gordeev, E. H. Haeusler, Proof Compression and NP Versus PSPACE, Studia Logica, vol. 107(1) (2019), pp. 55-83, DOI: https://doi.org/10.1007/ s11225-017-9773-5.

[3] L. Gordeev, E. H. Haeusler, Proof Compression and NP Versus PSPACE II, Bulletin of the Section of Logic, vol. 49(3) (2020), pp. 213-230, DOI: https://doi.org/10.18778/0138-0680.2020.16.

[4] E. H. Haeusler, Propositional Logics Complexity and the Sub-Formula Property, [in:] Proceedings of the Tenth International Workshop on Developments in Computational Models DCM (2014), arXiv:1504.01927.

[5] J. Hudelmaier, An $O(n \log n)$-space decision procedure for intuitionistic propositional logic, Journal of Logic and Computation, vol. 3 (1993), pp. 1-13, DOI: https://doi.org/10.1093/logcom/3.1.63.

[6] D. Prawitz, Natural deduction: A proof-theoretical study, Almqvist \& Wiksell (1965).

[7] R. Statman, Intuitionistic propositional logic is polynomial-space complete, Theoretical Computer Science, vol. 9 (1979), pp. 67-72, DOI: https: //doi.org/10.1016/0304-3975(79)90006-9.

\section{Lew Gordeev}

University of Tübingen

Department of Computer Science

Nedlitzer Str. 4a

14612 Falkensee, Germany

e-mail: lew.gordeew@uni-tuebingen.de

\section{E. Hermann Haeusler}

Pontificia Universidade Católica do Rio de Janeiro - RJ

Department of Informatics

Rua Marques de São Vicente, 224

Gávea, Rio de Janeiro, Brazil

e-mail: hermann@inf.puc-rio.br 